\title{
Influence of confining pressure on the mechanical and structural evolution of laboratory deformation bands
}

\author{
Karen Mair \\ Department of Earth Sciences, University of Liverpool, Liverpool, UK
}

Stephen Elphick and Ian Main

Department of Geology and Geophysics, University of Edinburgh, Edinburgh, UK

Received 23 August 2001; revised 2 April 2002; accepted 3 April 2002; published 24 May 2002.

[1] We present experimental observations of the influence of confining pressure on the mechanical behavior and structural style of damage in porous quartz rich sandstones. Large (100-mm diameter) samples of sandstone are deformed in a triaxial deformation apparatus, resulting in deformation expressed as pale interweaving bands of granulated material with finite shear offset and associated microcracking. The deformation fabrics evolve systematically from localized to more pervasive geometries with increasing confining pressure, associated with a systematic reduction in dynamic stress drop. The initial failure envelope is consistent with Mohr-Coulomb frictional behavior for all tests. These mechanical and structural observations confirm a gradual transition between brittle and semi-brittle behavior below the threshold for bulk cataclastic flow in a porous granular medium. Our experiments demonstrate that deformation band formation is strongly pressure-sensitive. The resulting structures are likely to have a strong anisotropic influence on permeability. INDEX TERMS: 8010 Structural Geology: Fractures and faults; 8020 Structural Geology: Mechanics; 5112 Physical Properties of Rocks: Microstructure; 5104 Physical Properties of Rocks: Fracture and flow

\section{Introduction}

[2] We present new experimental results showing the influence of confining pressure on the style of deformation bands produced in the laboratory. Deformation bands are small-displacement cataclastic faults occurring in highly porous quartz-rich sandstones that may have a significant impact on fluid flow. Field observations of deformation bands reveal them to be sites of concentrated damage where grain size, degree of sorting and porosity are all reduced with respect to the host rock [e.g., Aydin, 1978; Antonellini et al., 1994]. They commonly occur as complex anastamosing strands forming zones of damage [Aydin and Johnson, 1983].

[3] The mechanical behavior and structural expression of deformation in a porous sandstone may be influenced by many competing factors, including porosity, mineralogy, pore fluids, and confining pressure [Scott and Nielsen, 1991; Wong et al., 1997; Baud et al., 2000]. Recent experimental studies carried out at a wide range of conditions spanning the brittle, transitional, and plastic regimes have linked mechanical with microstructural [David et al., 2001; Klein et al., 2001] and hydraulic [David et al., 2001] observations. These studies give important insights into the mechanical behavior and failure mode of specific sandstones, but do not address the organization and evolution of multiple deformation bands. Recently, experiments carried out on large (100-mm diameter) core samples at constant confining

Copyright 2002 by the American Geophysical Union. 0094-8276/02/2001GL013964\$05.00 pressure have reproduced the major features of natural compound deformation bands, including the formation of increasing numbers of deformation bands as a direct function of accumulated strain [Mair et al., 2000].

[4] In this paper we extend the experimental method of Mair et al. [2000] to conditions of variable confining pressure to simulate the effect of burial depth on the structural style of deformation bands. We concentrate on experiments carried out in the brittle and semi-brittle regime and determine the specific characteristics of fault zone development by correlating mechanical and structural observations. We focus here on cataclastic deformation bands produced in high porosity quartz-rich sandstones, but note that other types of deformation bands (e.g., dilatant bands, compaction bands, clay smear bands) occur in rocks with different initial compositions, porosity or stress histories [Antonellini et al., 1994]. The results presented here have significant implications for the evolution of permeability in porous sandstones as a function of strain [Main et al., 2000] and confining pressure.

\section{Experimental Method}

[5] Initially intact cylindrical samples of Locharbriggs sandstone were deformed in a stiff large capacity triaxial apparatus, as described in Mair et al. [2000]. Locharbriggs sandstone has porosity $22.2 \%$, mean grain diameter $200 \mu \mathrm{m}$, and consists of quartz (88\%) and K-feldspar (6\%) clasts, with minor hematite grain coatings and silica cement. Samples were initially $100 \mathrm{~mm}$ in diameter, $230 \mathrm{~mm}$ in length with a minor sedimentary lamination perpendicular to length. A maximum compressive stress $\left(\sigma_{1}\right)$ is applied axially to the parallel ends of the sample by an external servo-controlled actuator. The minimum $\left(\sigma_{3}\right)$ and intermediate $\left(\sigma_{2}\right)$ compressive stresses are equal and are achieved by confining fluid pressurization of an impermeable soft jacket surrounding the sample sides.

[6] Pressure and displacement transducers continuously monitor the movement of loading pistons, the volume and pressure in confining fluid intensifiers, and the load at the sample. Axial stress and strain were computed from transducer measurements. Volumetric strain was calculated by monitoring changes in confining fluid volume required to maintain constant confining pressure. All experiments were conducted at a constant axial strain rate of $5 \times 10^{-6} \mathrm{~s}^{-1}$ and at room temperature and humidity with no pore fluid present. Tests underwent dynamic failure then post-failure deformation for a range of confining pressures with approximately constant ultimate strain. A structural analysis of samples was conducted following their removal from the testing apparatus.

\section{Experimental Results}

[7] Differential stress $\left(\sigma_{1}-\sigma_{3}\right)$ is plotted as a function of axial strain for a range of confining pressures in Figure 1a. Initial linear elastic behavior is followed, at $\sim 2 / 3$ of peak stress, by inelastic 

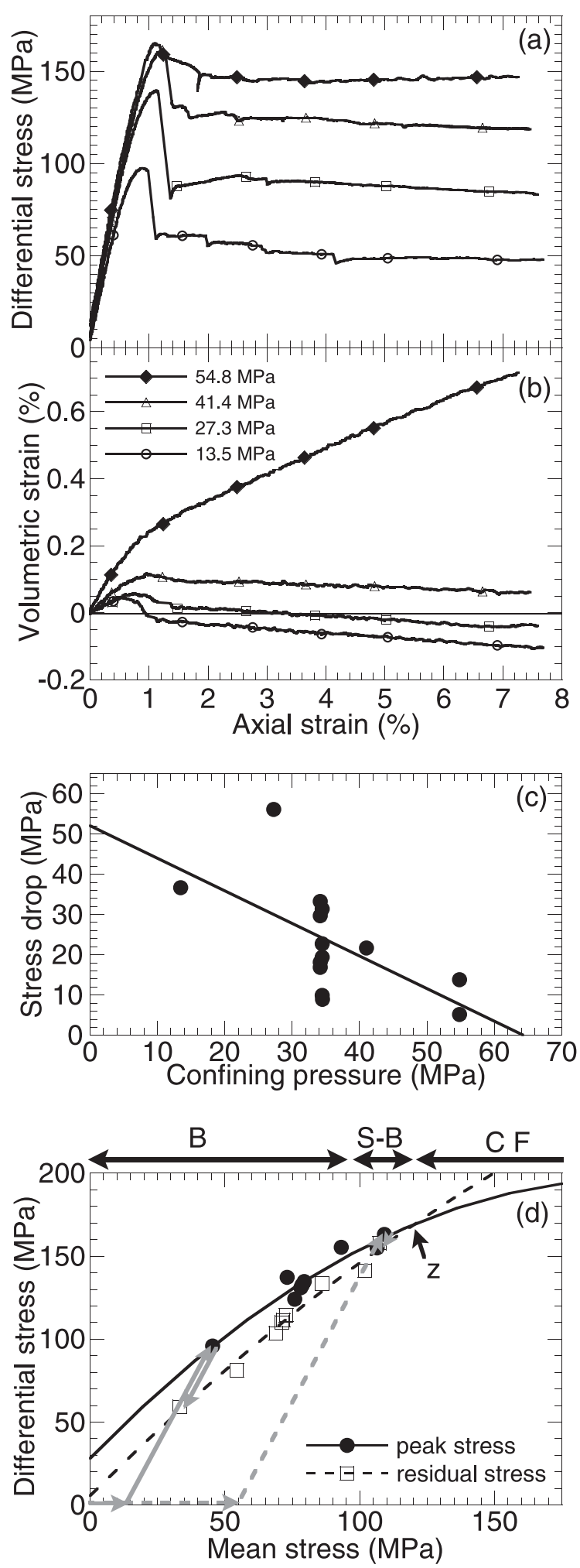

Figure 1. (a) Differential stress and (b) volumetric strain versus axial strain for confining pressures indicated. We assume compactive strains are positive. (c) Stress drop at failure as a function of confining pressure. A best-fit linear regression meets the $\mathrm{x}$-axis at $\sigma_{2}=\sigma_{3}=64 \mathrm{MPa}$. (d) p-q stress plot showing differential stress (q) versus mean stress (p). Peak and residual stress are fit by parabolas indicating failure and residual stress envelopes. Loading paths are indicated by gray arrows for $\sigma_{2}=\sigma_{3}=13.5 \mathrm{MPa}$ (solid) and $\sigma_{2}=\sigma_{3}$ $=54.8 \mathrm{MPa}$ (dotted). Brittle (B), semi-brittle (S-B) and cataclastic flow $(\mathrm{CF})$ regimes are shown. Zero stress drop is indicated $(\mathrm{Z})$. yield and strain hardening. The value of peak differential stress increases systematically with increasing confining pressure, and all samples undergo a finite stress drop after peak stress, typical of Mohr-Coulomb brittle failure [Paterson, 1978]. After failure, samples deform at an essentially constant stress that is a function of confining pressure.

[8] Volumetric strain curves (Figure 1b) indicate distinct phases of volume change correlating well with the stress-strain curves (Figure 1a). All experiments show initial compaction directly related to the applied confining pressure and subsequent changes of slope associated with sample yield and failure. After yield, the amount of dilatancy decreases systematically with confining pressure. Tests conducted at 13.5-41.4 MPa confining pressure show relative dilation after yield and further dilation at a reduced rate after sample failure, whereas at higher confining pressure (54.8 MPa) compaction dominates throughout loading.

[9] The stress drop associated with sample failure decreases systematically with increasing confining pressure (Figure 1c). If extrapolated, a linear trend predicts zero stress drop (a characteristic of plastic yield) at $\sigma_{2}=\sigma_{3} \approx 64 \mathrm{MPa}$. This may indicate a transition from brittle failure to bulk plastic flow [Bernabé and Brace, 1990]. To put our work in the context of recent studies [e.g., Wong et al., 1992] we plot differential stress $\left(\mathrm{q}=\sigma_{1}-\sigma_{3}\right)$ versus mean stress $\left(\mathrm{p}=\left(\sigma_{1}+\sigma_{2}+\sigma_{3}\right) / 3\right)$ as a standard $\mathrm{p}$-q stress plot, and include examples of the loading path (Figure 1d). Peak stress data are used to a construct the parabolic failure envelope indicating Mohr-Coulomb failure. Residual stress (post failure) is also fit by a parabolic envelope that intersects with the failure envelope at zero stress drop (predicted to occur at $p=120 \mathrm{MPa}$ ). All our data show a positive sensitivity of peak strength on mean stress, in contrast to the negative pressure dependence expected for compactive cataclastic flow [Wong et al., 1992].

[10] Optical examination of the deformed samples reveals a gradual progression in the style of macroscopic damage as a function of increasing confining pressure, from axial splitting, to localized deformation on sets of inclined sub-parallel shear bands, to multiple shear bands showing a range of strike orientations (Figure 2). Deformation fabrics are characterized by zones of pale anastamosing cataclastic fault strands $<1 \mathrm{~mm}$ wide (Figure 3), as in Mair et al. [2000], but show a striking systematic change in spatial organization of the bands with increasing confining pressure. The number of distinct bands and the maximum angle between $\sigma_{1}$ and shear bands both increase systematically with confining pressure in agreement with Bésuelle et al. [2000]. At low confining pressures deformation is localized onto a single discrete fracture zone. At the highest confining pressure deformation occurs in five major strike orientations, resulting in radial spokes traversing the sample and highlighting more distributed and isotropic deformation. Impor-
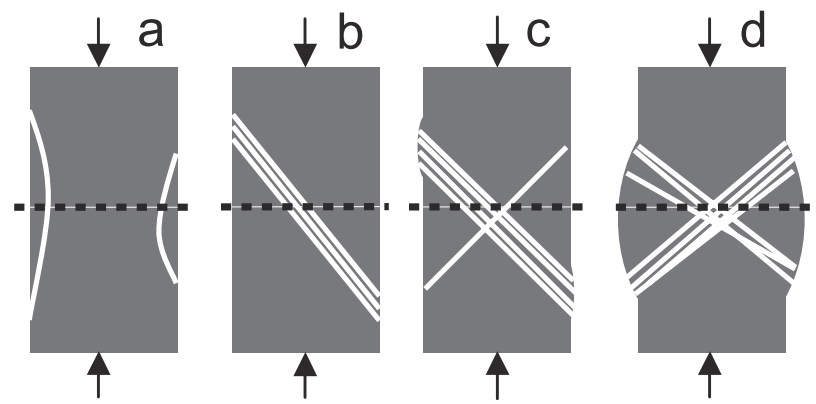

Figure 2. Schematic diagram showing the localization of damage in cylindrical samples as a function of confining pressures: (a) 13.5 MPa, (b) $27.3 \mathrm{MPa}$, (c) $41.4 \mathrm{MPa}$, and (d) $54.8 \mathrm{MPa}$. Arrows indicate maximum compressive stress $\left(\sigma_{1}\right)$ direction. Dotted lines indicate plane of Figure 3 photographs. Note in (d), shear bands are not necessarily conjugate but take a range of strikes. 


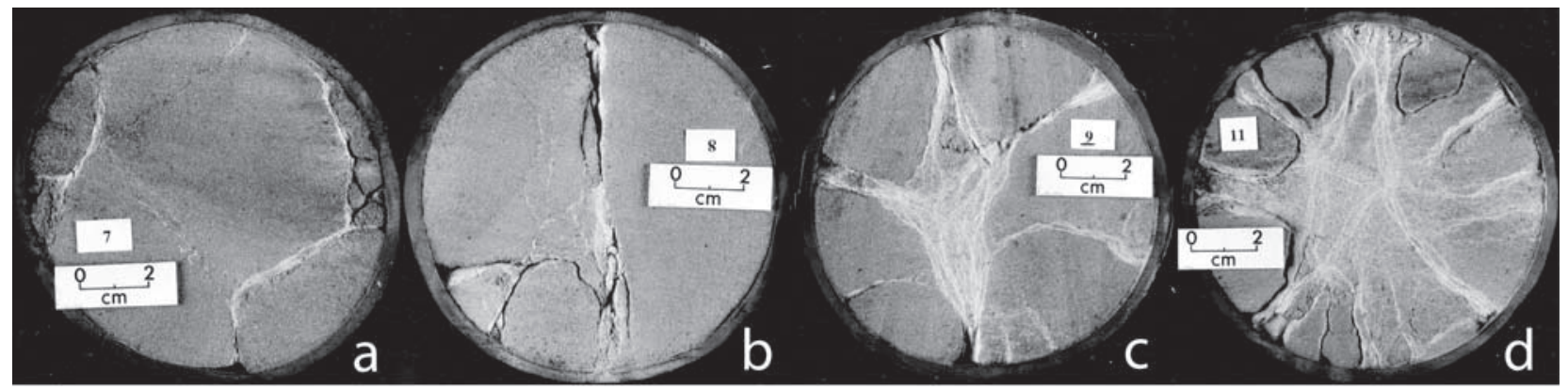

Figure 3. Photographs showing radial cross sections of Locharbriggs sandstone cores deformed to $7.8 \%$ axial strain under confining pressures of (a) $13.5 \mathrm{MPa}$, (b) $27.3 \mathrm{MPa}$, (c) $41.4 \mathrm{MPa}$, and (d) $54.8 \mathrm{MPa}$. Cores are cut perpendicular to at $\sigma_{1}$, as indicated on Figure 2, diameter is $\sim 100 \mathrm{~mm}$. Deformation is expressed as pale distinct strands whose spatial characteristics change systematically with increasing confining pressure.

tantly, the style of deformation is still in discrete wavy bands separated by host rock, and the individual units still involve localized damage rather than pervasive bulk cataclasis. Observations confirm that radial confining pressure $\left(\sigma_{3}=\sigma_{2}\right)$ has a strong control on the organization and mechanisms of damage, particularly at high confining pressure. Linear striations (or slickensides), in the direction of maximum dip, suggest shear movement along at least some of these features. However the relative timing of individual structures is not discernible.

[11] Photomicrographs of radial thin sections from a sample deformed at high confining pressure $(54.8 \mathrm{MPa})$ highlight the grain size reduction, poor sorting and porosity reduction typically found in cataclastic deformation bands (Figure 4). Isolated strands have little adjacent fracture (Figure 4a), whereas the region between two neighboring strands (Figure 4b) shows pervasive microfracture oriented sub-parallel to the strike of the gouge strands. Mair et al. [2000] indicate a microcrack anisotropy subparallel to $\sigma_{1}$ confirmed (but not shown) here. These cataclastic gouge strands and adjacent microcracks indicate that brittle grain scale processes such as microfracturing and frictional sliding are dominant irrespective of the macroscopic rheology under the conditions reported here.

[12] In summary, the individual deformation bands produced at low and high confining pressures have similar microscopic characteristics, but their macroscopic spatial organization is very distinct. The generic observation of a transition from localized to distributed damage with increasing pressure is typical of laboratory tests on crystalline rocks [Paterson, 1958] and smaller sandstone cores [e.g., Bernabé and Brace, 1990]. However, the specific development of increasingly isotropic and more numerous multiple bands in the semi-brittle field is novel.

\section{Discussion}

[13] Direct comparison of our results to other work is difficult since laboratory-induced compound deformation bands have rarely been reported elsewhere, although the structures correspond very well with field observations [Mair et al., 2000]. We observe increasing peak stress, decreasing stress drop, and an evolution from localized to distributed deformation with increasing confining pressure. This agrees well with laboratory studies of both shear and compaction deformation structures [Bésuelle et al., 2000; Wong et al., 2001, 1997, David et al., 2001]. Recent studies have highlighted a transitional (semi brittle) regime containing elements of brittle behavior, notably a finite stress drop and localized deformation features, in addition to plastic characteristics such as a distributed deformation style and shear-enhanced compaction. We interpret our mechanical data for Locharbriggs sandstone in this context as follows: a) brittle failure at $\sigma_{2}=\sigma_{3}=13.5-41.4$ $\operatorname{MPa}(\mathrm{p}=40-90 \mathrm{MPa}) ; \mathrm{b})$ a semi-brittle regime at $\sigma_{2}=\sigma_{3}=$
$54.8 \mathrm{MPa}(\mathrm{p}=90-120 \mathrm{MPa})$; and $\mathrm{c})$ predicted cataclastic flow at $\sigma_{2}=\sigma_{3}>64 \mathrm{MPa}(\mathrm{p}>120 \mathrm{MPa})$.

[14] Deformation mechanisms responsible for bulk behavior can be inferred from microstructural and mechanical data. Several
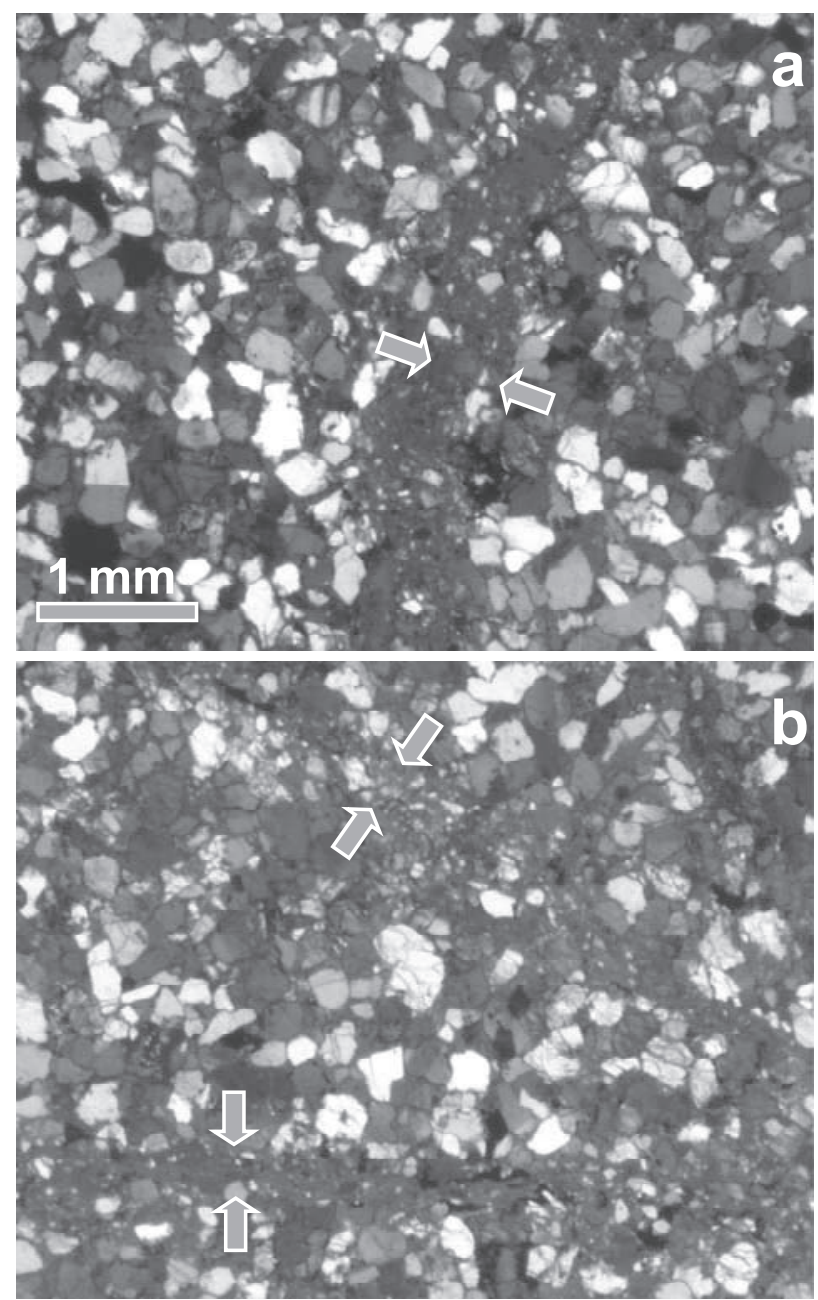

Figure 4. Photomicrographs of deformation bands in Figure 3d. Thin sections are cut perpendicular to $\sigma_{1}$. Individual deformation bands, indicated by arrows, show grain size reduction relative to host rock. (a) an isolated band shows a relative absence of associated microcracking, whereas (b) two proximal bands show an abundance of microcracking. 
microstructural features are common to all our experiments e.g. anisotropic intra-granular microcracking, cataclasis, grain size reduction and local porosity collapse. These observations suggest similar brittle micro-mechanisms operate at all confining pressures investigated here. However, at high confining pressure $\left(\sigma_{2}=\sigma_{3} \geq\right.$ $54.8 \mathrm{MPa}$ ) macroscopic dilatancy is suppressed, stress drop is reduced, and deformation becomes more distributed.

[15] These observations are entirely consistent with a transition from shear-enhanced dilatancy to shear enhanced compaction [Wong et al., 1997; Menéndez et al., 1996]. In their model for sandstone, dilatancy, prior to peak stress, is attributed to grain movements following intergranular microcracking as grain contacts rupture at high shear and low normal stresses. This mechanism (and hence dilatancy) is inhibited at high confining pressure because normal stresses are much higher and grain contacts cannot be easily ruptured. Near peak stress, intragranular cracking starts to operate. At low confining pressure this involves further shear movement and grain rearrangements, resulting in overall shearenhanced dilatancy. At high confining pressure, insitue grain fracture leads to local porosity collapse (shear-enhanced compaction) expressed macroscopically as bulk cataclastic flow. Our experiments interpreted as semi-brittle $\left(\sigma_{2}=\sigma_{3}=54.8 \mathrm{MPa}\right)$ indicate evidence for compaction mico-mechanisms (typical of cataclastic flow) operating within an overall brittle regime and leading to complex, semi-localized deformation structures.

\section{Conclusions}

[16] We demonstrate the importance of confining pressure on deformation band style produced in porous sandstone within an overall brittle regime, notably the transition from shear-enhanced dilatancy to compaction. We confirm the field-based hypothesis of Antonellini et al. [1994] that style, for a range of deformation bands, is strongly sensitive to burial depth, i.e. confining pressure or mean stress. Thus, any model for deformation band development will only apply within a finite depth range for a given loading path. However we note [e.g., Klein et al., 2001] that the processes leading to this type of deformation involve a crucial interplay between material properties and loading history. The results are likely to be specific to high porosity clean sandstones, and may not apply to materials with different clay content, grain cement or wider particle size distributions.

[17] Acknowledgments. K Mair is supported by a Royal Society Dorothy Hodgkin Fellowship. This work was partly funded by NERC studentship grant to K Mair. The authors acknowledge technical support from E Scholz and A Jackson and thank reviewers for comments that led to an improved paper.

\section{References}

Antonellini, M. A., A. Aydin, and D. D. Pollard, Microstructure of deformation bands in porous sandstones at Arches National Park, Utah, J. Struct. Geol., 16, 941-959, 1994.

Aydin, A., Small faults formed as deformation bands in sandstone, Pure and Appl. Geophys., 116, 913-930, 1978.

Aydin, A., and A. M. Johnson, Analysis of faulting in porous sandstones, J. Struct. Geol., 5, 19-31, 1983.

Baud, P., W. Zhu, and T.-F. Wong, Failure mode and weakening effect of water on sandstone, J. Geophys, Res., 105, 16,371-16,390, 2000.

Bernabé, Y., and W. F. Brace, Deformation and fracture of Berea sandstone, in The brittle-ductile transition in rock, edited by A. G. Duba et al., Geophys. Monogr., 56, AGU, Washington, D.C., 1990.

Bésuelle, P., J. Desrues, and S. Raynaud, Experimental characterization of the localization phenomenon inside a Vosges sandstone in a triaxial cell, Int. J. Rock Mech. Mining Sci., 37, 1223-1237, 2000.

David, C., B. Menéndez, W. Zhu, and T.-F. Wong, Mechanical compaction, microstructures and permeability evolution in sandstones, Phys. Chem. Earth (A), 26, 45-51, 2001.

Klein, E., P. Baud, T. Reuschlé, and T.-F. Wong, Mechanical behavior and failure mode of Bentheim sandstone under triaxial compression, Phys. Chem. Earth (A), 26, 21-25, 2001.

Main, I. G., O. Kwon, B. T. Ngwenya, and S. C. Elphick, Fault sealing during deformation band growth in sandstones, Geology, 28, 1131-1134, 2000.

Mair, K., I. Main, and S. Elphick, Sequential growth of deformation bands in the laboratory, J. Struct. Geol., 22, 25-42, 2000.

Menéndez, B., W. Zhu, and T.-F. Wong, Micromechanics of brittle faulting and cataclastic flow in Berea sandstone, J. Struct. Geol., 18, 1-16, 1996.

Paterson, M. S., Experimental deformation and faulting in Wombeyan marble, Bull. Geol. Soc. Am., 69, 465-476, 1958.

Paterson, M. S., Experimental rock deformation - the brittle field, SpringerVerlag, Berlin, 1978.

Scott, T. E., and K. C. Nielsen, The effect of porosity on the brittle ductile transition in sandstones, J. Geophys. Res., 96, 405-414, 1991.

Wong, T.-F., C. David, and W. Zhu, The transition from brittle faulting to cataclastic flow in porous sandstones: Mechanical deformation, J. Geophys. Res., 102, 3009-3025, 1997.

Wong, T.-F., H. Szeto, and J. Zhang, Effect of loading path and porosity on the failure mode of porous rocks, Appl. Mech. Rev., 45, 281-293, 1992.

Wong, T.-F., P. Baud, and E. Klein, Localized failure modes in a compactant porous rock, Geophysical Research Letters, 28, 2521-2524, 2001.

K. Mair, Department of Earth Sciences, University of Liverpool, Liverpool, L69 3GP, UK. (k.mair@liverpool.ac.uk)

I. Main and S. Elphick, Department of Geology and Geophysics, University of Edinburgh, Edinburgh, EH9 3JW, UK. 